\title{
EDITORIAL
}

\section{BE PREPARED}

\author{
A recent case of an uncooperative patient with tuberculosis has highlighted the need for \\ public-health legislation in England and Wales to be updated.
}

It has been estimated that, untreated, an individual with infectious tuberculosis (TB) can infect 10-15 other individuals every year. But in England and Wales, doctors have no authority to give compulsory treatment for TB, or in fact any infectious disease.

In one particular case that has been widely reported in the UK this month, it is believed that at least 12 individuals have contracted TB from one infected person who is aware that he has the disease but has so far refused to complete a full course of treatment. The individual in question was diagnosed with TB in 2002 and is believed to have taken antibiotics only for short periods when his symptoms were most acute.

The ability of the local health authority, or the criminal authorities, to intervene has been restricted by the available legislation in England and Wales. Under Sections 35, 37 and 38 of the Public Health (Control of Diseases) Act 1984 , magistrates can order that an individual who poses a serious infectious risk to others should undergo compulsory medical examination and can be removed to, and detained in, a hospital, but there is no provision for compulsory treatment. In the case in question, a magistrate did order the individual to attend hospital, but as he refused treatment once he was there, he was subsequently released.

Although refusal of treatment for an infectious disease such as TB is a rare event, this case clearly has many interesting implications, not just for TB control. Specifically, it highlights the need for the Public Health Act, some of which still dates from Victorian times, to be updated to reflect the current threats from emerging and re-emerging infectious diseases.

Calls for changes to the Act were initially made when the first cases of HIV infection occurred in the UK. However, the first - and last - broad consultation exercise was held at the end of the 1980s, and although there was a clear consensus that the legislation should be updated, so far no changes have been made. This is despite the fact that, over the past 20 years, the infectious disease landscape has changed dramatically. The last new additions to the list of notifiable diseases in England and Wales (meningococcal septicaemia without meningitis, mumps and rubella) were made in 1988, bringing the total number of notifiable diseases to 30 . Notable omissions include variant Creutzfeldt-Jacob disease, Legionnaire's disease and HIV. Although voluntary notification of these conditions by doctors is excellent and therefore no change in reporting would be expected if they were placed on the list, their omission provides a useful illustration of how outdated the legislation has become.

In the Department of Health's contingency plan to tackle the latest perceived threat — an influenza pandemic - there is some discussion of social distancing measures to reduce morbidity and/or contain the spread of infection, including voluntary home isolation of infected individuals and voluntary quarantine of known contacts. However, any revision of the Public Health Act to allow compulsory quarantine measures to be implemented, for example, is clearly not on the agenda. The report states that "Voluntary co-operation with recommended measures would be sought. Mandatory quarantine and curfews are generally not considered necessary and are not currently covered by public health legislation." Additionally, the Chief Medical Officer was recently quoted as saying that in the event of a pandemic, social intervention measures such as closing schools and cinemas will be kept "very much in reserve".

Any public health response to a serious infectiousdisease outbreak must of course be proportionate, but it should also be aided where possible by the available legislation. In the event of an emergency such as an influenza pandemic, widespread non-compliance with voluntary quarantine measures is obviously very much a worst-case scenario. But the best emergency plans take all eventualities into account and, given the potential consequences of this worst-case scenario, it would surely be prudent to have the necessary legislation in place to allow health officials to react effectively in the unlikely event that it occurs.

In the 'post-SARS' era, many countries have been evaluating their public health legislation to assess how it would withstand an emergency challenge - perhaps it is time for England and Wales to do the same? 